\title{
Lower sexual satisfaction in women with polycystic ovary syndrome and metabolic syndrome
}

\author{
Małgorzata Kałużna1 ${ }^{\mathbb{D}}$, Agnieszka Nomejko ${ }^{\mathbb{D} 2}$, Aleksandra Słowińska² ${ }^{\mathbb{D}}$, Katarzyna Wachowiak-Ochmańska³ \\ Katarzyna Pikosz ${ }^{4}$, Katarzyna Ziemnicka'D and Marek Ruchała ${ }^{1}$
}

${ }^{1}$ Department of Endocrinology, Metabolism and Internal Diseases, Poznan University of Medical Sciences, Poznan, Poland

${ }^{2}$ Institute of Psychology, Faculty of Pedagogical and Historical Sciences, University of Wrocław, Wrocław, Poland ${ }^{3}$ Endocrinology, Metabolism and Internal Diseases Ward, Heliodor Swiecicki University Hospital, Poznan, Poland

${ }^{4}$ Department of Pharmacognosy, Poznan University of Medical Sciences, Poznan, Poland

Correspondence should be addressed to M Kałużna: kaluznama@gmail.com

\begin{abstract}
Background: Polycystic ovary syndrome (PCOS) is a multi-symptom disorder linked with a range of metabolic and hormonal disturbances. Psychological and sexual aspects of PCOS also need to be considered.

Objective of the study: This study aimed to assess sexual satisfaction (SS) in PCOS patients and eumenorrheic controls (CON). The relationships between SS, depressive symptoms, health-related quality of life (HRQoL), and hormonal and metabolic profiles were evaluated.

Methods: In this study, 190 patients with PCOS (mean age $26.34 \pm 5.47$ years) and 197 age-matched CON (mean age $27.12 \pm 4.97$ years) were enrolled. All subjects completed Polish version of the Sexual Satisfaction Questionnaire (SSQ), WHO Quality of Life-BREF (WHOQOL-BREF), and the Center for Epidemiologic Studies Depression Scale-Revised (CESD-R) questionnaire. Fasting blood samples were collected to assess hormonal, lipid, and glucose profiles. Anthropometric measures were collected. Metabolic syndrome (MS) was evaluated according to the IDF-AHA/NHLBI criteria.

Results: Patients with PCOS and MS had lower SS vs non-MS-PCOS. There were no significant differences in the level of SS, presence of depressive symptoms, or HRQoL between PCOS and CON $(P>0.05)$. Negative correlations were found between the SS level and BMI, waist circumference, and waist-to-height ratio in PCOS women. However, overweight or obese PCOS women did not differ in SS levels vs normal-weight PCOS patients. The social dimension of WHOQOL-BREF was the only significant predictor of SS in PCOS patients.

Conclusions: SS in PCOS women appears to be undisturbed. However, MS in PCOS patients could negatively influence SS. The level of SS should be assessed in PCOS women, especially if MS is present.
\end{abstract}
Key Words
- polycystic ovary syndrome (PCOS)
- health-related quality of life (HRQoL)
- WHO quality of life-BREF (WHOQOL-BREF)
- sexual satisfaction
- sexual satisfaction questionnaire (SSQ)

\section{Introduction}

Polycystic ovary syndrome (PCOS) is a common condition, affecting up to $20 \%$ of reproductive-aged women (1, 2). PCOS is associated with significant hormonal, reproductive, metabolic, and psychological concerns. PCOS is characterized by substantial clinical heterogeneity and high comorbidity of psychosomatic diseases (3). https://ec.bioscientifica.com https://doi.org/10.1530/EC-21-0257

(c) 2021 The authors Published by Bioscientifica Ltd
Endocrine Connections (2021) 10, 1035-1044

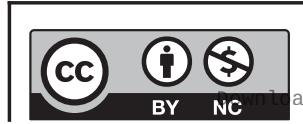

This work is licensed under a Creative Commons Attribution-NonCommercial 4.0 International License. ded from Bioscientifica.com at 04/26/2023 10:27:0๑AM 
The clinical phenotype of PCOS varies by ethnicity and age-related changes. Psychological features such as anxiety, depression, body image distress are often observed in PCOS women $(4,5,6)$. Irregular menstrual cycles, hirsutism, acne, and infertility may aggravate stress and psychological disorders $(7,8)$.

PCOS patients deal with biopsychosocial changes that may affect sexual functioning and decrease sexual satisfaction (SS) $(9,10,11)$. For example, objective PCOS characteristics (parity, excessive weight, and current unfulfilled wish to conceive) and PCOS-related concerns (women's infertility-related and acne-related problems) can be associated with lower SS (12). However, Silva et al. found that $81.3 \%$ of PCOS women were satisfied with their sexual life (13). Moreover, Zhao et al.'s meta-analysis suggested no direct association between PCOS and the risk of female sexual dysfunction (FSD) (14). Stevanovic et al. also did not show differences in sexual function between Europid PCOS and healthy women in research on the QoL using the 50-item health-related quality of life questionnaire for polycystic ovary syndrome (PCOSQ-50) (including 7 items on sexual function) $(15,16)$.

Sexual health is an integral part of health understood holistically, and the relationship between sexual health and the remaining components of health is bidirectional. The level of SS - a subjective dimension of health - is related to the health-related quality of life (HRQoL) and mental and physical health (17). Sexual dissatisfaction and psychological issues are often under-appreciated in patients with chronic diseases and can go unrecognized $(18,19)$. Identification and treatment of psychological disorders in women with PCOS particularly during critical ages such as adolescence are part of current practice for diagnosis and management of the PCOS by specialists across Europe (20). Although international evidence-based guidelines for the assessment and management of PCOS and a position statement from the European Society of Endocrinology (ESE) address PCOS's psychological features and recommend measuring heath-related QoL in all PCOS patients, limited data on FSD are available in PCOS to date $(6,21)$. Moreover, validated questionnaires have not been used often to evaluate SS.

\section{Aim}

This study aimed to screen SS and psychological conditions in PCOS women in comparison to eumenorrheic controls. The relationship of depressive symptoms, HRQoL, and clinical phenotype of PCOS (clinical and biochemical hyperandrogenism (HA), simple and central obesity, insulin resistance (IR), lipid disturbances, metabolic syndrome (MS)) with SS was evaluated.

\section{Methods}

In this study, 190 patients with PCOS (mean age $26.34 \pm 5.47$ years) and 197 age-matched control subjects (CON) (mean age $27.12 \pm 4.97$ years) were recruited. The PCOS patients were diagnosed according to the Rotterdam criteria and the latest international evidencebased guidelines for the assessment and management of PCOS $(21,22)$. Following these recommendations, PCOS features were defined as HA, oligoovulation, and polycystic ovarian morphology (PCOM) in ultrasound. The presence of $\geq 2$ out of the following three features: oligoovulation or anovulation, clinical and/or biochemical HA, or PCOM determined the presence of PCOS $(21,22)$. Biochemical HA was defined as total testosterone $(\mathrm{T})>2.67 \mathrm{nmol} / \mathrm{L}$ and/or free testosterone index $(\mathrm{FTI})>5.5(23,24,25)$. A volume above $10 \mathrm{~cm}^{3}$ and more than 20 follicles sized 2-9 $\mathrm{mm}$ were criteria for PCOM (21). The Ferriman-Gallwey scale of 8 points as the cut-off value was used to assess hirsutism.

A CON group consisted of eumenorrheic healthy individuals without reported problems concerning endocrine disorders, sexual development, and maturation. PCOS and CON women were excluded if they had severe psychiatric disorders (schizophrenia, bipolar disorder, severe depression), diabetes, severe liver or kidney disease, the use of oral contraceptive or anti-androgen therapy in the last 3 months or current pregnancy or diagnosed and/ or treated infertility. Sociodemographic characteristics were comparable between PCOS and CON groups. Information on anthropometric parameters was collected from all participants. Measurement of biochemical and hormonal parameters was performed for the entire PCOS group and about a quarter of the CON participants $(n=49)$.

The weight, height, waist circumference (WC), and hip circumference (HC) were measured according to the World Health Organization (WHO) recommendations. Women with $\mathrm{BMI} \geq 25 \mathrm{~kg} / \mathrm{m}^{2}$ were defined as obese (26). Waistto-height ratio (WHtR) was calculated by dividing WC by height. Subjects with $\mathrm{WHtR} \geq 0.5$ were defined as centrally obese (26). Measurements of systolic blood pressure (SBP) and diastolic blood pressure (DBP) were performed in the sitting position, using a validated automated oscillometric monitor, following standards of BP measurements.

Serum biochemical measurements (glucose, insulin, sex hormone-binding globulin (SHBG)) and serum hormone

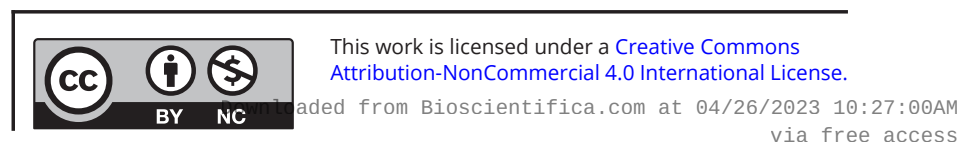


levels (follicle-stimulating hormone (FSH), luteinizing hormone (LH), estradiol (E2), T, DHEA sulfate (DHEA-S)) were measured in patients (on days 3-5 of the menstrual cycle or after a progestin-induced withdrawal bleeding) with the Cobas 6000 equipment (Roche Diagnostics), using kits provided by the manufacturer. Hexokinase method (Roche Diagnostics) with a variation (CV) coefficient of $\leq 3 \%$ for serum glucose was applied. Anti-Müllerian hormone (AMH) was measured using the Elecsys ${ }^{\oplus} \mathrm{AMH}$ assay by Roche Diagnostics (detection limit: $0.071 \mathrm{pmol} / \mathrm{L}$ ); measuring range: $0.071-164.2 \mathrm{pmol} / \mathrm{L}$, intermediate precision: 2.5-3.9\% CV). Total cholesterol (TC), high-density lipoprotein cholesterol (HDL-C), and triglycerides (TG) were measured by the enzymatic colorimetric method. Lowdensity lipoprotein cholesterol (LDL-C) was estimated by the Friedewald formula: LDL-C-C =TC-HDL-C-VLDL-C(TG/5). The homeostatic model assessment for insulin resistance (HOMA-IR) and free androgen index (FAI) were calculated: fasting insulin $(\mathrm{U} / \mathrm{L}) \times$ fasting glucose $(\mathrm{mg} / \mathrm{dL}) / 405$, and $(100 \times \mathrm{T}(\mathrm{nmol} / \mathrm{L})) / \mathrm{SHBG}(\mathrm{nmol} / \mathrm{L}))$, respectively (27). Insulin resistance (IR) was defined as HOMA-IR > 2.5. Metabolic syndrome (MS) was assessed according to the IDF-AHA/ NHLBI criteria (28). A set of three scales were collated and administered to both groups. The survey forms included the Polish version of the Sexual Satisfaction Questionnaire (SSQ), the WHO Quality of Life-BREF (WHOQOL-BREF), and the Center for Epidemiologic Studies Depression ScaleRevised (CESD-R) questionnaire. Consent has been obtained from each patient after full explanation of the purpose and nature of all procedures used. The study was approved by the Board of Bioethics of Poznan University of Medical Science $(552 / 16 ; 986 / 17)$.

\section{Sexual satisfaction questionnaire (SSQ)}

The SSQ is a valid and reliable psychometric method to assess the respondents' cognitive and emotional approach to their own sexual life and evaluate the level of SS (29). This questionnaire comprises ten items scored on a 1-4 Likert scale. The respondents were divided into categories and subgroups with low, medium, or high results based on the total score (sum of 10 items). For females, the total score was characterized as follows: 10-25 low; 26-31 medium; $32-40$ high result (29).

\section{WHO quality of life-BREF (WHOQOL-BREF)}

WHOQOL-BREF is a global cross-culturally comparable HRQoL assessment instrument. The WHOQOL-BREF questionnaire consists of 26 questions, including physical health, psychological health, social relationships, and environment. The first two of the 26 items could be examined separately. Question 1 asks about an individual's global QoL rate, and question 2 asks how individuals perceive their health status. The WHOQOLBREF instrument is a shorter adaptation of the original questionnaire - WHOQOL (30). Question number 21 (How satisfied are you with your sex life?) was excluded from the analyses.

\section{The center for epidemiologic studies depression scale-revised (CESD-R)}

The Polish version of the CESD-R scale was used as a psychometric evaluation of depression. This self-reported scale, developed by L S Radloff and updated by W Eaton, serves as a screening tool to evaluate the presence of affective symptoms, especially depression, in populationbased samples. The CESD-R consists of 20 clear and understandable statements linked with the mood and behavior during the last 2 weeks (31).

\section{Statistical analysis}

Statistical analysis of the data was performed using SPSS version 21.0 (SPSS, Inc.). The normality of data distribution was verified with the Shapiro-Wilk test or the KolmogorovSmirnov test with Lilleforce significance correction, depending on the sample size. Moreover, skewness and kurtosis of the variables were controlled. Analyses were conducted on raw data from questionnaires. Continuous variables were compared using the Student's $t$-test for parametric data and the Mann-Whitney test for nonparametric data. Categorical data were compared using the Chi-squared test. Pearson linear correlation was used for correlation analysis between two continuous variables with normal or close to a normal distribution, and Spearman's rank correlation for continuous non-normal variables. Linear regression analysis was performed for continuous, normally distributed variables. All tests were considered statistically significant at $P<0.05$.

\section{Results}

\section{Descriptive statistics and intergroup differences}

In the first step, the significance of differences between the PCOS and CON groups in all variables was checked. The results of the analyses were provided in Tables 1 and 2 .

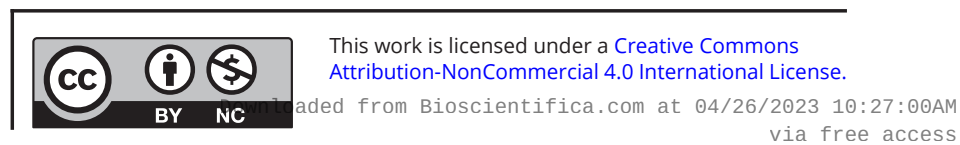


Table 1 Results of tests describing the significance of differences in the control and polycystic ovary syndrome groups.

\begin{tabular}{|c|c|c|c|}
\hline & CON & Pcos & \\
\hline & $M( \pm$ S.D. $)$ & $M( \pm$ S.D. $)$ & $P$-value \\
\hline \multicolumn{4}{|l|}{ Anthropometric parameters } \\
\hline Age & $27.12(4.97)$ & $26.34(5.47)$ & 0.14 \\
\hline Body mass (kg) & $64.47(14.65)$ & $70.39(16.41)$ & 0.001 \\
\hline Height (cm) & $166.83(6.45)$ & $166.33(5.84)$ & 0.43 \\
\hline BMI $\left(\mathrm{kg} / \mathrm{m}^{2}\right)$ & $23.14(4.93)$ & $25.46(5.91)$ & $<0.001$ \\
\hline $\mathrm{WC}(\mathrm{cm})$ & $77.06(13.21)$ & $82.03(13.86)$ & $<0.001$ \\
\hline WHtR & $0.46(.09)$ & $0.49(.09)$ & $<0.001$ \\
\hline SBP & $118.95(11.50)$ & $119.49(12.69)$ & 0.90 \\
\hline DBP & $74.32(9.75)$ & $74.95(8.93)$ & 0.95 \\
\hline \multicolumn{4}{|l|}{ Biochemical parameters } \\
\hline $\mathrm{TC}(\mathrm{mg} / \mathrm{dL})$ & $168.18(28.00)$ & $172.56(34.02)$ & 0.73 \\
\hline LDL-C (mg/dL) & $89.07(29.67)$ & $92.35(37.14)$ & 0.91 \\
\hline TG (mg/dL) & $79.27(27.88)$ & $94.36(61.75)$ & 0.54 \\
\hline $\mathrm{HDL}-\mathrm{C}(\mathrm{mg} / \mathrm{dL})$ & $63.45(12.81)$ & $63.73(16.29)$ & 0.84 \\
\hline Glucose (mg/dL) & $88.94(7.62)$ & 90.17 (18.09) & 0.95 \\
\hline Insulin (mIU/mL) & $10.86(7.41)$ & $11.41(8.22)$ & 0.93 \\
\hline HOMA-IR & $2.47(1.87)$ & $2.76(3.62)$ & 0.83 \\
\hline \multicolumn{4}{|l|}{ Hormonal parameters } \\
\hline $\mathrm{FSH}(\mathrm{mlU} / \mathrm{mL})$ & $5.23(2.21)$ & $5.97(2.00)$ & 0.01 \\
\hline $\mathrm{LH}(\mathrm{mlU} / \mathrm{mL})$ & $7.81(7.03)$ & $11.38(8.34)$ & $<0.001$ \\
\hline DHEAS $(\mu \mathrm{g} / \mathrm{dL})$ & $289.72(105.36)$ & $325.51(134.92)$ & 0.12 \\
\hline $\mathrm{E} 2(\mathrm{pg} / \mathrm{mL})$ & $81.28(66.45)$ & $64.72(62.99$ & 0.10 \\
\hline $\mathrm{T}(\mathrm{nmol} / \mathrm{L})$ & $1.28(.58)$ & $1.77(.73)$ & $<0.001$ \\
\hline SHBG (nmol/L) & $75.30(51.66)$ & $61.71(36.70)$ & 0.31 \\
\hline $\mathrm{FTI}(\%)$ & $2.49(1.95)$ & $4.06(3.45)$ & $<0.001$ \\
\hline $\mathrm{TSH}(\mathrm{mlU} / \mathrm{mL})$ & $2.12(1.23)$ & $2.16(1.19)$ & 0.65 \\
\hline $\mathrm{AMH}(\mathrm{pmol} / \mathrm{L})$ & $3.50(1.22)$ & $9.21(5.74)$ & $<0.001$ \\
\hline \multicolumn{4}{|l|}{ Psychological characteristics } \\
\hline SSQ total score & $31.48(6.22)$ & $31.43(6.24)$ & 0.94 \\
\hline Depressive symptoms & $14.44(11.93)$ & $13.39(10.63)$ & 0.62 \\
\hline WHO QoL physical health & $21.23(3.37)$ & $21.15(3.09)$ & 0.81 \\
\hline WHO QoL psychological health & $21.00(3.07)$ & $20.43(3.15)$ & 0.08 \\
\hline WHO QoL social relationships & $7.78(1.61)$ & $7.80(1.46)$ & 0.66 \\
\hline WHO QoL environment & $29.77(3.90)$ & $29.58(4.37)$ & 0.08 \\
\hline WHO QoL WHO question 1 & $4.05(0.66)$ & $4.00(0.64)$ & 0.42 \\
\hline WHO QoL WHO question 2 & $3.69(0.84)$ & $3.30(0.86)$ & $<0.001$ \\
\hline
\end{tabular}

$\mathrm{AMH}$, anti-Müllerian hormone; CON, control subjects; DBP, diastolic blood pressure; ; E2, estradiol; FSH, follicle-stimulating hormone; FTI, free testosterone index; HDL, high-density lipoprotein cholesterol; HOMA-IR, homeostasis model assessment for insulin resistance index; LDL-C, low-density lipoprotein cholesterol; LH, luteinizing hormone; M ( \pm S.D.), mean \pm standard deviation; PCOS, polycystic ovary syndrome patients; QoL, quality of life; SBP, systolic blood pressure; SHBG, sex hormone-binding globulin; SSQ, sexual satisfaction questionnaire; T, total testosterone; TC, total cholesterol; TG, triglycerides; TG/HDL-C, the ratio of triglycerides to HDL-cholesterol; TSH, thyroid-stimulating hormone; WC, waist circumference; WHtR, waist-to-height ratio.

Regarding the basic research question, the results indicate that there were no differences in the level of SS between the PCOS and CON groups $(P=0.94)$. In the PCOS group, $18.4 \%$ ( $n=35)$ of participants had a low level of SS, $21.1 \%(n=40)$ a moderate level, and $60.5 \%(n=115)$ a high level of SS. In the CON group, the levels of SS were 19.8\% $(n=39), 19.8 \%(n=39)$, and 60.4\% (n=119), respectively.

For the anthropometric characteristics, significant intergroup differences were found. PCOS patients were characterized by higher body mass, BMI, WC, and WHtR than CON subjects $(P<0.05$, Table 1$)$. Moreover, there were significantly more overweight, obese, and central obese women in the PCOS than in the CON group $(P<0.05$, Table 2$)$.

There were no significant differences between the groups in biochemical parameters $(P>0.05$, Table 1$)$ or in the incidence of MS and IR $(P>0.05$, Table 2$)$. The PCOS patients had a higher level of FSH, LH, T, FTI, and AMH $(P<0.05$, Table 1$)$. Moreover, acne and hirsutism were more frequent in the PCOS than in the CON group $(P<0.05$, Table 2$)$.

There were no significant differences between the groups for most psychological variables. All participants were characterized by a similar level of depressive https://ec.bioscientifica.com https://doi.org/10.1530/EC-21-0257 (c) 2021 The authors Published by Bioscientifica Ltd
This work is licensed under a Creative Commons Attribution-NonCommercial 4.0 International License. ded from Bioscientifica.com at 04/26/2023 10:27:0๑AM via free access 
Table 2 Clinical characteristics of the control and polycystic ovary syndrome groups.

\begin{tabular}{|c|c|c|c|}
\hline & CON & Pcos & \\
\hline & $n(\%)$ & $n(\%)$ & $P$-value \\
\hline Acne & $12(22.6)$ & 95 (56.9) & $<0.001$ \\
\hline Hirsutism & $12(22.2)$ & $91(54.5)$ & $<0.001$ \\
\hline PCOM & $1(2.5)$ & $126(81.8)$ & $<0.001$ \\
\hline Overweight & $47(24.6)$ & $76(40.6)$ & $<0.001$ \\
\hline Obesity & $17(8.9)$ & $38(20.3)$ & 0.002 \\
\hline Central obesity & $38(24.5)$ & 66 (37.3) & 0.01 \\
\hline MS & $3(6.4)$ & $26(16)$ & 0.09 \\
\hline IR & $17(32.1)$ & $61(37)$ & 0.52 \\
\hline
\end{tabular}

CON, control subjects; IR, insulin resistance; MS, metabolic syndrome; PCOM, polycystic ovarian morphology at ultrasound; PCOS, polycystic ovary syndrome patients; insulin resistance, homeostasis model assessment for insulin resistance index $>2.5$.

symptoms and HRQoL in the health, psychological, social, and environmental domains. Only in question number 2 of WHOQOL-BREF there was a difference - the CON subjects were more satisfied with their overall health than the PCOS subjects $(P<0.05$, Table 1$)$.

\section{Anthropometric correlates of sexual satisfaction}

The correlation analysis between SS and anthropometric parameters did not reveal any significant relationships in CON subjects. In contrast, significant (but weak) correlations were observed in the PCOS group between SSQ score and anthropometry. Higher body mass, WC, BMI, and WHtR were associated with the lower SS level $(P<0.05$, Table $3)$. However, the analysis of differences between the groups distinguished based on the criterion of overweight, simple, and central obesity conducted in the PCOS group showed that these variables did not differentiate SS levels (Table 4).

Table 3 Correlation analysis between sexual satisfaction and anthropometric parameters in the control and polycystic ovary syndrome groups.

\begin{tabular}{|c|c|c|}
\hline SSQ total score \& & CON & Pcos \\
\hline Age & -0.03 & -0.09 \\
\hline Weight & $-0.12^{a}$ & $-0.16^{a, b}$ \\
\hline Height & -0.09 & 0.08 \\
\hline BMI & $-0.10^{a}$ & $-0.18^{a, b}$ \\
\hline WC & $-0.07^{a}$ & $-0.20^{c}$ \\
\hline WHtR & 0.06 & $-0.16^{a, b}$ \\
\hline SBP & 0.04 & -0.03 \\
\hline DBP & 0.21 & -0.09 \\
\hline
\end{tabular}

Bold values were significant at $P<0.05$.

aSpearman's correlation coefficient, unmarked Pearson's correlation coefficient; ${ }^{\mathrm{b}} P<0.05 ;{ }^{\mathrm{c}} P<0.01$

CON, control subjects; DBP, diastolic blood pressure; PCOS, polycystic ovary syndrome patients; SBP, systolic blood pressure.
Table 4 Sexual satisfaction in the subpopulations of the polycystic ovary syndrome patients.

\begin{tabular}{|c|c|c|c|}
\hline & Present & Absent & \\
\hline Criterion & $M( \pm$ S.D. $)$ & $M( \pm$ S.D. $)$ & P-value \\
\hline Acne & 31.62 (6.17) & $31.51(6.48)$ & 0.94 \\
\hline Hirsutism & $31.23(6.43)$ & $31.99(6.12)$ & 0.43 \\
\hline $\mathrm{PCOM}^{\mathrm{a}}$ & 31.75 (6.27) & $31.16(5.66)$ & 0.40 \\
\hline Overweight & $30.26(6.88)$ & $32.37(5.63)$ & 0.06 \\
\hline Obesity & 30.09 (7.78) & $31.88(5.75)$ & 0.42 \\
\hline Central obesity & 30.92 (6.91) & $32.15(5.85)$ & 0.37 \\
\hline MS & $28.10(8.37)$ & $32.16(5.78)$ & 0.04 \\
\hline IR & $31.48(7.58)$ & $31.63(5.48)$ & 0.30 \\
\hline
\end{tabular}

aPCOM, polycystic ovary morphology, volume and the morphology of each ovary, setting the threshold at $10 \mathrm{~cm}^{3}$ for increased ovarian volume and 20 for the 2-9 $\mathrm{mm}$ follicles.

$\mathrm{IR}$, insulin resistance; MS, metabolic syndrome.

\section{Biochemical correlates of sexual satisfaction}

The correlation analysis results found no relationship between SS and biochemical parameters in either group (Table 5). Within the PCOS group, there was also no difference in SS between patients with or without IR (Table 4). PCOS patients with MS had a lower SS level than those without MS $(P<0.05$, Table 4$)$. However, it should be noted that the results of the analysis carried out on data from PCOS and CON groups do not allow to conclude that the presence of MS differentiates the level of women's SS $(P>0.05)$.

\section{Hormonal correlates of sexual satisfaction}

The correlation analysis between the SS level and hormonal parameters showed that both groups' hormone concentrations were not related to perceived SS (Table 6).

Table 5 Correlation analysis between sexual satisfaction and biochemical parameters in the control and polycystic ovary syndrome groups.

\begin{tabular}{|c|c|c|}
\hline SSQ total score \& & CON & PCOS \\
\hline $\mathrm{TC}$ & -0.19 & $-0.08^{a}$ \\
\hline LDL-C & -0.15 & $-0.05^{a}$ \\
\hline TG & 0.19 & $0.06^{a}$ \\
\hline HDL-C & -0.13 & 0.08 \\
\hline Glucose & -0.12 & $0.05^{a}$ \\
\hline Insulin & $0.03^{a}$ & $0.005^{a}$ \\
\hline HOMA-IR & $0.04^{a}$ & $0.02^{a}$ \\
\hline
\end{tabular}

$P$-values for all correlation coefficients $>0.05$.

aSpearman's correlation coefficient; unmarked Pearson's correlation coefficient.

CON, control subjects; HDL-C, high-density lipoprotein cholesterol; HOMA-IR, homeostasis model assessment for insulin resistance index; LDL-C, low-density lipoprotein cholesterol; PCOS, polycystic ovary syndrome patients; TC, total cholesterol; TG, triglycerides.

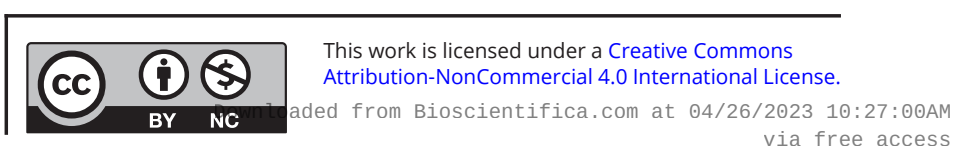


Table 6 Correlation analysis between sexual satisfaction and hormonal parameters in the control and polycystic ovary syndrome groups.

\begin{tabular}{l}
\hline sSQ total score \& \\
\hline FSH $(\mathrm{mlU} / \mathrm{mL})$ \\
LH $(\mathrm{mlU} / \mathrm{mL})$ \\
DHEAS $(\mu \mathrm{g} / \mathrm{dL})$ \\
E2 $(\mathrm{pg} / \mathrm{mL})$ \\
T $(\mathrm{nmol} / \mathrm{L})$ \\
SHBG $(\mathrm{nmol} / \mathrm{L})$ \\
FTI $(\%)$ \\
TSH $(\mathrm{mIU} / \mathrm{mL})$ \\
AMH
\end{tabular}

\begin{tabular}{c}
\hline CON \\
\hline-0.13 \\
$-0.19^{\mathrm{a}}$ \\
-0.11 \\
$-0.06^{\mathrm{a}}$ \\
$0.10^{\mathrm{a}}$ \\
$-0.15^{\mathrm{a}}$ \\
$0.27^{\mathrm{a}}$ \\
$0.21^{\mathrm{a}}$ \\
$0.003^{\mathrm{a}}$ \\
\hline
\end{tabular}

\begin{tabular}{c}
\hline PCOS \\
\hline-0.11 \\
$-0.10^{a}$ \\
0.03 \\
$0.12^{a}$ \\
$0.05^{a}$ \\
$0.06^{a}$ \\
$-0.03^{a}$ \\
$-0.02^{a}$ \\
$0.19^{a}$ \\
\hline
\end{tabular}

$P$-values for all correlation coefficients $>0.05$.

aSpearman's correlation coefficient; unmarked Pearson's correlation coefficient.

$\mathrm{AMH}$, anti-Müllerian hormone; CON, control subjects; E2, estradiol; FSH, follicle-stimulating hormone; FTI, free testosterone index; $\mathrm{LH}$, luteinizing hormone; PCOS, polycystic ovary syndrome patients; SHBG, sex hormonebinding globulin; SSQ, sexual satisfaction questionnaire; T, total testosterone; TSH, thyroid-stimulating hormone.

Moreover, in the PCOS group, acne and hirsutism were also not associated with the SS level (Table 4).

\section{Psychological correlates of sexual satisfaction}

Regarding psychological parameters, the correlation analysis revealed many relationships with the level of SS in both groups (Table 7). A higher level of depressive symptoms was associated with lower SS $(P<0.05)$ and this correlation was higher in the CON than in the PCOS $(z=2.1$, $P=0.04)$. A higher level of SS was related to the HRQoL in all four dimensions (physical health, psychological health, social relationships, and environment) and the general assessment of the HRQoL (question 1 of WHOQOL-BREF) and health satisfaction (question 2 of WHOQOL-BREF;

Table 7 Correlation analysis between sexual satisfaction and psychological parameters in the control and polycystic ovary syndrome subjects.

\begin{tabular}{l}
\hline SSQ total score \& \\
\hline Depressive symptoms \\
WHO QOL physical health \\
WHO QOL psychological health \\
WHO QOL social relationships \\
WHO QOL environment \\
WHO QOL WHO question 1 \\
WHO QOL WHO question 2
\end{tabular}

\begin{tabular}{c}
\hline CON \\
\hline$-0.41^{a, b}$ \\
$0.33^{b}$ \\
$0.43^{b}$ \\
$0.39^{b}$ \\
$0.28^{b}$ \\
$0.34^{a, b}$ \\
$0.19^{c}$ \\
\hline
\end{tabular}

\begin{tabular}{c}
\hline PCOS \\
\hline$-0.21^{a, c}$ \\
$0.18^{c}$ \\
$0.25^{c}$ \\
$0.27^{b}$ \\
$0.21^{c}$ \\
$0.18^{a, d}$ \\
$0.19^{c}$ \\
\hline
\end{tabular}

$P$-values for all correlation coefficients $<0.05$.

aspearman's correlation coefficient, unmarked Pearson's correlation coefficient; ${ }^{b} P<0.001 ; c P<0.01 ; ;$ d $P<0.05$.

CON, control subjects; PCOS, polycystic ovary syndrome patients; WHO QoL, the quality of life-based on WHO quality of life-BREF questionnaire.
$P<0.05)$. The relationship between SS and HRQoL in the psychological dimension was more robust in the CON than in the PCOS subjects $(z=1.97, P<0.05)$. The remaining correlations were characterized by a similar strength in both groups.

In the next step, enter method regression analysis was carried out to determine which HRQoL dimensions are the best predictors of SS. Biochemical and hormonal parameters were not included as predictors due to the lack of correlation with SS. The analysis was performed separately for either group. HRQoL in the social dimension was the only significant predictor of SS in the PCOS group $(P<0.05)$, explaining $8 \%$ of its variance (Table 8$)$. In the CON, predictors of SS were both the HRQoL's social and psychological dimensions $(P<0.05)$. These variables explained $21 \%$ of the variance in SS.

\section{Discussion}

The main findings of this study are as follows: (i) PCOS women with MS were characterized by lower SS vs non-MSPCOS. (ii) The level of SS, presence of depressive symptoms, and HRQoL are comparable in the whole PCOS and CON groups. (iii) Biochemical and clinical hyperandrogenism, simple and central obesity, PCOM, IR level, and lipid concentrations seem not to significantly independently affect SS in PCOS patients. (iv) The level of SS correlates with assessed psychological variables. The social dimension of WHOQOL-BREF was the only significant predictor of SS in PCOS. In contrast, both the social and psychological dimensions of WHOQOL-BREF were significant predictors of SS in CON.

Surveyed women, both PCOS and CON, declared a medium level of SS. Over $60 \%$ of PCOS women were highly satisfied with their sex life (32). The research results are consistent with the observations of Silva et al. of high SS in women with PCOS (13). Similarly, Ercan et al. observed a comparable prevalence of FSD in the PCOS vs controls (25\% vs 19\%; $P=0.54$ ) (33). A systemic review and metaanalysis by Zhao et al., including 1163 women with PCOS, revealed no differences in total female sexual function index (FSFI) scores between PCOS vs controls (14). Other studies, employing a $100 \mathrm{~mm}$ visual analog scale, suggested that PCOS severely limits SS $(11,34)$. In the assessment of the so-called objective SS (including e.g. proper genital function, experiencing orgasm, etc.), women receive lower results than assessing subjective SS (35, 36, 37). Moreover, the feeling of satisfaction with sexual life and objective sexual functioning are two different issues (37).

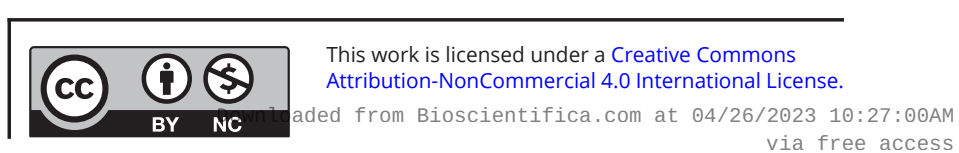


Table 8 Regression models predicting sexual satisfaction in the control and PCOS groups.

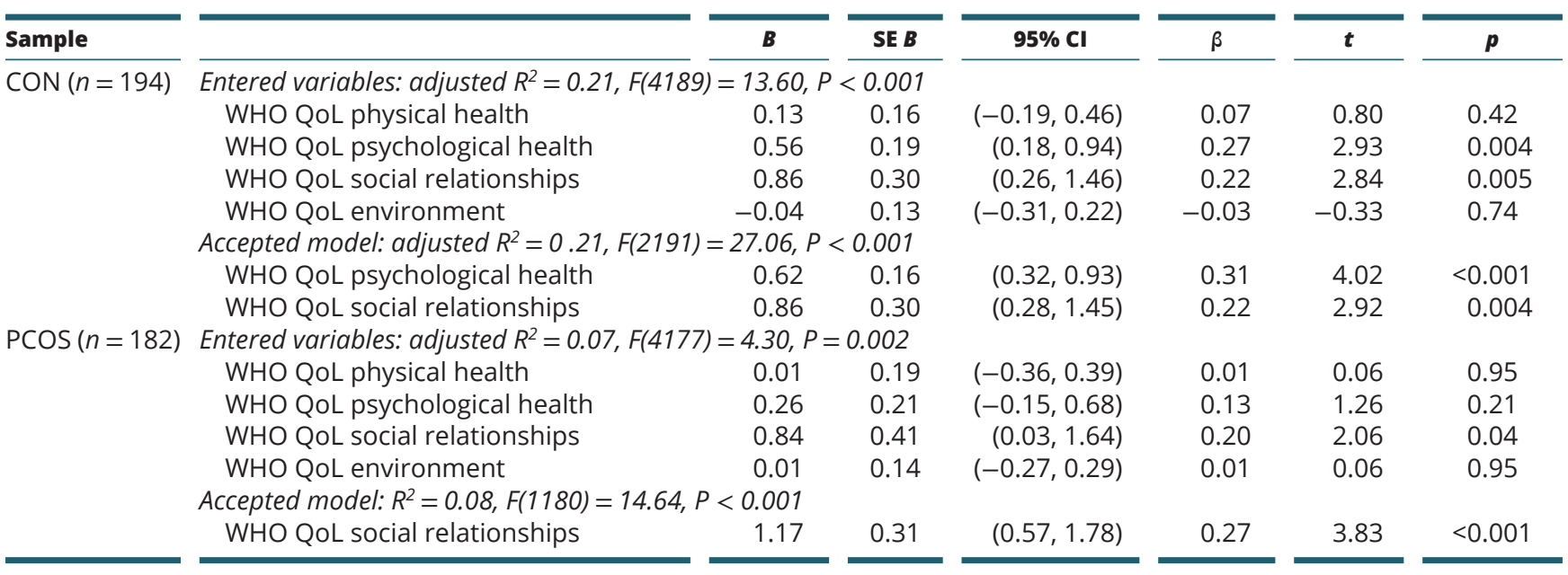

A range of psychological factors, mostly the quality of a close relationship and the context for sexual activity, have a more substantial influence on SS in women vs men $(36,38,39)$. Cultural and ethnic factors could impact SS, as well (17).

Our results suggest a negative association of metabolic syndrome with sexual satisfaction in PCOS. PCOS women are at increased risk of MS, including visceral obesity, IR, and lipid disturbances $(40,41)$. The link between SS and MS has been studied extensively $(42,43,44)$. Esposito et al. noted lower scores on the FSFI (predominantly in the SS domain) in premenopausal women with MS vs general female population (42). MS has also been associated with lower SS in postmenopausal women $(43,44)$. The current results suggest no correlations between SS and individual components of MS, except for WC. However, the constellation of visceral adiposity, increased BP, and lipid and glucose metabolism disturbances comprising the MS could have a negative impact on SS in PCOS. Of note, MS-PCOS women were characterized by moderate SS, whereas non-MS-PCOS women had high SS in SSQ in the current study. If lower SS in PCOS women with MS has a psychological basis or somatic reasons (vascular or nervous problems) or both of these, it should be further studied.

Obesity is an indispensable feature of about 50\% of PCOS women (45). The parameters of simple and visceral obesity correlated negatively with SS level in PCOS women $(P<0.05$, Table 3$)$. Interestingly, no correlations were found between SS and obesity parameters in the CON ( $P>0.05$, Table 3). Notwithstanding, overweight, obese, or central obese PCOS women did not differ in SS level vs normal-weight PCOS patients (Table 4). Similar results were obtained by Aloulou et al., who observed that BMI had no impact on the SS and frequency of sexual intercourse in PCOS (9). More studies are needed to clarify the possible complex link between excess fat mass, and PCOS patient sexuality.

Lipidand glucosedisorders areincreased independently in PCOS (46). No significant association between lipid and glucose profile and SS was present in the current study (Table 5). On the contrary, lower SS was observed in patients with dyslipidemia in a study by Esposito et al. (47). A higher prevalence of FSD was observed in women with hyperlipidemia and low HDL vs normolipidemic females $(47,48,49)$. In a study by Baldassarre et al., dyslipidemia was independently associated with FSD (49). It may be the case that the severity and type of hyperlipidemia matter. Current study found no detectable effect of isolated hyperlipidemia on SS in PCOS.

Predictable hormonal alterations in PCOS patients were noted in the study, but in the correlation analysis, no relationship was found between the total SSQ scores and female sex hormones, androgens, and TSH. Ercan et al. did not note statistical correlations between the hormonal profile and sexual function, except for $\mathrm{LH}$, free testosterone, T, insulin, and HOMA-IR (33). However, in a covariance analysis with adjustment for hormone levels, no effect of PCOS on FSFI score was reported (33). Similarly, Veras et al. noted a negative correlation between the scores of the Arizona Sexual Experience Scale and the levels of T, LH, and DHEAS (50). However, again, no impairment in sexual function was shown in PCOS patients (50). Veras et al. hypothesized that in PCOS women, hyperandrogenemia could protect the sexual functions (50). The impact of
This work is licensed under a Creative Commons Attribution-NonCommercial 4.0 International License. ed from Bioscientifica.com at 04/26/2023 10:27:00AM 
androgens on female sexual function is complex, and conflicting data are available $(51,52,53,54)$. Subsequent studies on the complex role of different androgens on female sexuality are needed.

The present study proved the significant relationship between the HRQoL and SS. The HRQoL was comparable between PCOS and CON women. Previous studies have shown that PCOS causes a significant reduction in HRQoL (34). Research in PCOS women showed a considerable influence of BMI and hirsutism on HRQoL (55). PCOSrelated symptoms (acne, obesity, hirsutism) can induce depression and limit HRQoL (6). The current research results are consistent with a study by Silva et al. - SS of PCOS women was not influenced by BMI or hirsutism (13). Many researchers point out the bidirectional relationship between self-esteem, sense of attractiveness, and sexual functioning $(17,57,58)$. In the current research, there was no statistically significant difference in PCOS and CON responses to the WHOQOL-BREF question 11 (Are you able to accept your bodily appearance?) and question 19 (How satisfied are you with yourself?). More studies are needed to clarify this topic in PCOS.

Current study revealed that social dimension was the only predictor of SS in PCOS, whereas in CON, both social and psychological dimensions were significant SS predictors. In a meta-analysis by Pastoor et al., the high impact for the social effect of appearance and sexual attractiveness were shown in PCOS women (3). This result confirms the importance of social and relational support and couple's counseling for the sexual functioning of PCOS women. The current study supports the thesis on the relationship between SS and the presence of depressive symptoms $(59,60)$. However, the incidence of depressive symptoms was not significantly different between PCOS and $\mathrm{CON}$ women. The contrary findings on the prevalence of depressive syndromes in PCOS could be due to ethnic diversity, differences in the previous patients' medical experiences, the distinction in psychological support, and various HRQoL evaluation tools $(4,5)$.

\section{Limitations}

First, a broader assessment of SS based on factors related to sexual functioning and satisfaction with intimate relationship should be conducted in the future. Next, the measurement of biochemical and hormonal parameters was not performed for the entire CON group. Thirdly, CESD-R is not a typical clinical diagnostic scale, but its usefulness in studying the prevalence of depressive syndrome in the general population was proven (31).

\section{Conclusions}

Metabolic syndrome in PCOS women could negatively influence SS. Screening of SS should be a component of PCOS management. SS is closely associated with HRQoL and the presence of depressive symptoms. The social dimension of WHOQOL-BREF was the only significant predictor of SS in PCOS patients. Women diagnosed with PCOS, satisfied with social support and personal relationships can lead a highly satisfying sexual life.

\section{Declaration of interest}

The authors declare that there is no conflict of interest that could be perceived as prejudicing the impartiality of the research reported.

\section{Funding}

This work did not receive any specific grant from any funding agency in the public, commercial or not-for-profit sector.

\section{References}

1 March WA, Moore VM, Willson KJ, Phillips DI, Norman RJ \& Davies MJ. The prevalence of polycystic ovary syndrome in a community sample assessed under contrasting diagnostic criteria. Human Reproduction 201025 544-551. (https://doi.org/10.1093/ humrep/dep399)

2 Bozdag G, Mumusoglu S, Zengin D, Karabulut E \& Yildiz BO. The prevalence and phenotypic features of polycystic ovary syndrome: a systematic review and meta-analysis. Human Reproduction 201631 2841-2855. (https://doi.org/10.1093/humrep/dew218)

3 Pastoor H, Timman R, de Klerk C, Bramer WM, Laan ET \& Laven JS. Sexual function in women with polycystic ovary syndrome: a systematic review and meta-analysis. Reproductive Biomedicine Online 201837 750-760. (https://doi.org/10.1016/j.rbmo.2018.09.010)

4 Chaudhari AP, Mazumdar K \& Mehta PD. Anxiety, depression, and quality of life in women with polycystic ovarian syndrome. Indian Journal of Psychological Medicine 201840 239-246. (https://doi. org/10.4103/IJPSYM.IJPSYM_561_17)

5 Prathap A, Subhalakshmi TP \& Varghese PJ. A cross-sectional study on the proportion of anxiety and depression and determinants of quality of life in polycystic ovarian disease. Indian Journal of Psychological Medicine 201840 257-262. (https://doi.org/10.4103/IJPSYM. IJPSYM_221_17)

6 Conway G, Dewailly D, Diamanti-Kandarakis E, Escobar-Morreale HF Franks S, Gambineri A, Kelestimur F, Macut D, Micic D, Pasquali R, et al. The polycystic ovary syndrome: a position statement from the European Society of Endocrinology. European Journal of Endocrinology 2014171 P1-29. (https://doi.org/10.1530/EJE-14-0253)

7 Ekback MP, Lindberg M, Benzein E \& Arestedt K. Health-related quality of life, depression and anxiety correlate with the degree of hirsutism. Dermatology 2013227 278-284. (https://doi. org/10.1159/000355356)

8 Deeks AA, Gibson-Helm ME \& Teede HJ. Anxiety and depression in polycystic ovary syndrome: a comprehensive investigation. Fertility and Sterility 201093 2421-2423. (https://doi.org/10.1016/j. fertnstert.2009.09.018)

9 Aloulou J, Halouani N, Charfeddine F, Mseddi N, Charfi N, Abid M \& Amami O. P-1334-marital sexual satisfaction in women with

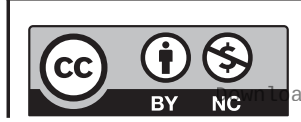

This work is licensed under a Creative Commons Attribution-NonCommercial 4.0 International License. ded from Bioscientifica com at 04/26/2023 10:27:00AM 
polycystic ovary syndrome. European Psychiatry 201227 1-1. (https:// doi.org/10.1016/S0924-9338(12)75501-3)

10 Mansson M, Norstrom K, Holte J, Landin-Wilhelmsen K, Dahlgren E \& Landen M. Sexuality and psychological wellbeing in women with polycystic ovary syndrome compared with healthy controls. European Journal of Obstetrics, Gynecology, and Reproductive Biology 2011155 161-165. (https://doi.org/10.1016/j.ejogrb.2010.12.012)

11 Thannickal A, Brutocao C, Alsawas M, Morrow A, Zaiem F, Murad MH $\&$ Javed Chattha A. Eating, sleeping and sexual function disorders in women with polycystic ovary syndrome (PCOS): a systematic review and meta-analysis. Clinical Endocrinology 202092 338-349. (https:// doi.org/10.1111/cen.14153)

12 De Frene V, Verhofstadt L, Loeys T, Stuyver I, Buysse A \& De Sutter P. Sexual and relational satisfaction in couples where the woman has polycystic ovary syndrome: a dyadic analysis. Human Reproduction 201530 625-631. (https://doi.org/10.1093/humrep/deu342)

13 Silva JS, Fonseca AM, Bagnoli VR, Cavalcanti AL, Soares Jr JM \& Baracat EC. Sexuality in women with polycystic ovary syndrome: a pilot study. Einstein 20108 397-403. (https://doi.org/10.1590/S167945082010AO1836)

14 Zhao S, Wang J, Xie Q, Luo L, Zhu Z, Liu Y, Luo J \& Zhao Z. Is polycystic ovary syndrome associated with risk of female sexual dysfunction? A systematic review and meta-analysis. Reproductive Biomedicine Online 201938 979-989. (https://doi.org/10.1016/j.rbmo.2018.11.030)

15 Nasiri-Amiri F, Ramezani Tehrani F, Simbar M, Montazeri A \& Mohammadpour RA. Health-related quality of life questionnaire for polycystic ovary syndrome (PCOSQ-50): development and psychometric properties. Quality of Life Research 201625 1791-1801. (https://doi.org/10.1007/s11136-016-1232-7)

16 Stevanovic D, Bozic-Antic I, Stanojlovic O, Vojnovic Milutinovic D, Bjekic-Macut J, Jancic J \& Macut D. Health-related quality of life questionnaire for polycystic ovary syndrome (PCOSQ-50): a psychometric study with the Serbian version. Women and Health 2019 59 1015-1025. (https://doi.org/10.1080/03630242.2019.1587664)

17 Frissora CL \& Koch KL. Symptom overlap and comorbidity of irritable bowel syndrome with other conditions. Current Gastroenterology Reports 20057 264-271. (https://doi.org/10.1007/s11894-005-0018-9)

18 Jones GL, Hall JM, Lashen HL, Balen AH \& Ledger WL. Health-related quality of life among adolescents with polycystic ovary syndrome. Journal of Obstetric, Gynecologic, and Neonatal Nursing 201140 577-588. (https://doi.org/10.1111/j.1552-6909.2011.01279.x)

19 Crete J \& Adamshick P. Managing polycystic ovary syndrome: what our patients are telling us. Journal of Holistic Nursing 201129 256-266. (https://doi.org/10.1177/0898010111398660)

20 Conway G, Dewailly D, Diamanti-Kandarakis E, Escobar-Morreale HF, Franks S, Gambineri A, Kelestimur F, Macut D, Micic D, Pasquali R, et al. European Survey of Diagnosis and Management of the polycystic ovary syndrome: results of the ESE PCOS Special Interest Group's Questionnaire. European Journal of Endocrinology $2014171489-498$. (https://doi.org/10.1530/EJE-14-0252)

21 Teede HJ, Misso ML, Costello MF, Dokras A, Laven J, Moran L, Piltonen T, Norman RJ \& International PCOS Network. Recommendations from the international evidence-based guideline for the assessment and management of polycystic ovary syndrome. Human Reproduction 201833 1602-1618. (https://doi.org/10.1093/ humrep/dey256)

22 Rotterdam ESHRE/ASRM-Sponsored PCOS Consensus Workshop Group. Revised 2003 consensus on diagnostic criteria and long-term health risks related to polycystic ovary syndrome. Fertility and Sterility 200481 19-25. (https://doi.org/10.1016/j.fertnstert.2003.10.004)

23 Lerchbaum E, Schwetz V, Rabe T, Giuliani A \& Obermayer-Pietsch B. Hyperandrogenemia in polycystic ovary syndrome: exploration of the role of free testosterone and androstenedione in metabolic phenotype. PLOS ONE 20149 e108263. (https://doi.org/10.1371/journal. pone.0108263)
24 Saxena U, Ramani M \& Singh P. Role of AMH as diagnostic tool for polycystic ovarian syndrome. Journal of Obstetrics and Gynaecology of India 201868 117-122. (https://doi.org/10.1007/s13224-017-1066-4)

25 Kaluzna M, Czlapka-Matyasik M, Wachowiak-Ochmanska K, Moczko J, Kaczmarek J, Janicki A, Piatek K, Ruchala M \& Ziemnicka K. Effect of central obesity and hyperandrogenism on selected inflammatory markers in patients with PCOS: a WHtR-matched case-control study. Journal of Clinical Medicine 20209 3024. (https://doi.org/10.3390/ jcm9093024)

26 Ching YK, Chin YS, Appukutty M, Gan WY, Ramanchadran V \& Chan YM. Prevalence of metabolic syndrome and its associated factors among vegetarians in Malaysia. International Journal of Environmental Research and Public Health 201815 2031. (https://doi.org/10.3390/ ijerph15092031)

27 Matthews DR, Hosker JP, Rudenski AS, Naylor BA, Treacher DF \& Turner RC. Homeostasis model assessment: insulin resistance and beta-cell function from fasting plasma glucose and insulin concentrations in man. Diabetologia 198528 412-419. (https://doi. org/10.1007/BF00280883)

28 Alberti KG, Eckel RH, Grundy SM, Zimmet PZ, Cleeman JI, Donato KA, Fruchart JC, James WP, Loria CM, Smith Jr SC, et al. Harmonizing the metabolic syndrome: a joint interim statement of the International Diabetes Federation Task Force on Epidemiology and Prevention; National Heart, Lung, and Blood Institute; American Heart Association; World Heart Federation; International Atherosclerosis Society; and International Association for the Study of Obesity. Circulation 2009120 1640-1645. (https://doi.org/10.1161/ CIRCULATIONAHA.109.192644)

29 Nomejko AD-Z \& Dolińska-Zygmunt G. The sexual satisfaction questionnaire - psychometric properties. Polish Journal of Applied Psychology 201412 105-112. (https://doi.org/10.1515/pjap-2015-0017)

30 Kyle UG, Schutz Y, Dupertuis YM \& Pichard C. Body composition interpretation. Contributions of the fat-free mass index and the body fat mass index. Nutrition 200319 597-604. (https://doi.org/10.1016/ s0899-9007(03)00061-3)

31 Tripathy P, Sahu A, Sahu M \& Nagy A. Ultrasonographic evaluation of intra-abdominal fat distribution and study of its influence on subclinical atherosclerosis in women with polycystic ovarian syndrome. European Journal of Obstetrics, Gynecology, and Reproductive Biology 2017217 18-22. (https://doi.org/10.1016/j.ejogrb.2017.08.011)

32 Nomejko A \& Dolińśka-Zygmunt G. Sexual satisfaction of women and men in early, middle and late adulthood - psychosocial determinants: who and when does sex bring joy? In Sexual satisfaction of women and men, ch 4, pp 83-86.Kraków, Poland: Impuls, 2019.

33 Ercan CM, Coksuer H, Aydogan U, Alanbay I, Keskin U, Karasahin KE \& Baser I. Sexual dysfunction assessment and hormonal correlations in patients with polycystic ovary syndrome. International Journal of Impotence Research 201325 127-132. (https://doi.org/10.1038/ ijir.2013.2)

34 Elsenbruch S, Hahn S, Kowalsky D, Offner AH, Schedlowski M, Mann K \& Janssen OE. Quality of life, psychosocial well-being, and sexual satisfaction in women with polycystic ovary syndrome. Journal of Clinical Endocrinology and Metabolism 200388 5801-5807. (https:// doi.org/10.1210/jc.2003-030562)

35 Kaygorodova IA \& Dzyuba EV. New information on the distribution pattern of Acanthobdella peledina (Annelida, Acanthobdellida) in Eastern Siberia. Zootaxa 20184399 123-126. (https://doi.org/10.11646/ zootaxa.4399.1.8)

36 Basson R. The female sexual response: a different model. Journal of Sex and Marital Therapy 200026 51-65. (https://doi. org/10.1080/009262300278641)

37 Ferenidou F, Kapoteli V, Moisidis K, Koutsogiannis I, Giakoumelos A \& Hatzichristou D. Presence of a sexual problem may not affect women's satisfaction from their sexual function. Journal of Sexual Medicine 2008 5 631-639. (https://doi.org/10.1111/j.1743-6109.2007.00644.x) https://ec.bioscientifica.com https://doi.org/10.1530/EC-21-0257 (c) 2021 The authors Published by Bioscientifica Ltd

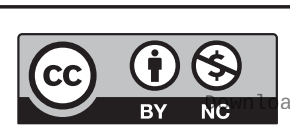

This work is licensed under a Creative Commons Attribution-NonCommercial 4.0 International License. ded from Bioscientifica.com at 04/26/2023 10:27:0๑AM 
38 Nobre PJ \& Pinto-Gouveia J. Emotions during sexual activity: differences between sexually functional and dysfunctional men and women. Archives of Sexual Behavior 200635 491-499. (https://doi. org/10.1007/s10508-006-9047-1)

39 Dundon CM \& Rellini AH. More than sexual function: predictors of sexual satisfaction in a sample of women age 40-70. Journal of Sexual Medicine 20107 896-904. (https://doi.org/10.1111/j.17436109.2009.01557.x)

$40 \mathrm{Mu}$ L, Zhao Y, Li R, Lai Y \& Qiao J. Metabolic characteristics of normal weight central obesity phenotype polycystic ovary syndrome women: a large-scale national epidemiological survey. Reproductive Biomedicine Online 201837 498-504. (https://doi.org/10.1016/j.rbmo.2018.08.007)

41 Behboudi-Gandevani S, Ramezani Tehrani F, Hosseinpanah F, Khalili D, Cheraghi L, Kazemijaliseh H \& Azizi F. Cardiometabolic risks in polycystic ovary syndrome: long-term population-based follow-up study. Fertility and Sterility 2018110 1377-1386. (https://doi. org/10.1016/j.fertnstert.2018.08.046)

42 Esposito K, Ciotola M, Marfella R, Di Tommaso D, Cobellis L \& Giugliano D. The metabolic syndrome: a cause of sexual dysfunction in women. International Journal of Impotence Research 200517 224-226. (https://doi.org/10.1038/sj.ijir.3901310)

43 Trompeter SE, Bettencourt R \& Barrett-Connor E. Metabolic syndrome and sexual function in postmenopausal women. American Journal of Medicine 2016129 1270.e1-1277.e1. (https://doi.org/10.1016/j. amimed.2016.03.039)

44 Silva GM, Lima SM \& Moraes JC. Evaluation of sexual function in postmenopause women with metabolic syndrome. Revista Brasileira de Ginecologia e Obstetricia 201335 301-308. (https://doi.org/10.1590/ s0100-72032013000700004)

45 Sam S. Obesity and polycystic ovary syndrome. Obesity Management 20073 69-73. (https://doi.org/10.1089/obe.2007.0019)

46 Diamanti-Kandarakis E. Role of obesity and adiposity in polycystic ovary syndrome. International Journal of Obesity 200731 (Supplement 2) S8-S13; discussion S31-S12. (https://doi.org/10.1038/sj.ijo.0803730)

47 Esposito K, Ciotola M, Maiorino MI, Giugliano F, Autorino R, De Sio M, Cozzolino D, Saccomanno F \& Giugliano D. Hyperlipidemia and sexual function in premenopausal women. Journal of Sexual Medicine 20096 1696-1703. (https://doi.org/10.1111/j.1743-6109.2009.01284.x)

48 Martelli V, Valisella S, Moscatiello S, Matteucci C, Lantadilla C, Costantino A, Pelusi G, Marchesini G \& Meriggiola MC. Prevalence of sexual dysfunction among postmenopausal women with and without metabolic syndrome. Journal of Sexual Medicine 20129 434-441. (https://doi.org/10.1111/j.1743-6109.2011.02517.x)

49 Baldassarre M, Alvisi S, Mancini I, Moscatiello S, Marchesini G, Seracchioli R \& Meriggiola MC. Impaired lipid profile is a risk factor for the development of sexual dysfunction in women. Journal of Sexual Medicine 201613 46-54. (https://doi.org/10.1016/j.jsxm.2015.11.005)
50 Veras AB, Bruno RV, de Avila MA \& Nardi AE. Sexual dysfunction in patients with polycystic ovary syndrome: clinical and hormonal correlations. Comprehensive Psychiatry 201152 486-489. (https://doi. org/10.1016/j.comppsych.2010.10.013)

51 Kepczynska-Nyk A, Kurylowicz A, Nowak A, Bednarczuk T \& Ambroziak U. Sexual function in women with androgen excess disorders: classic forms of congenital adrenal hyperplasia and polycystic ovary syndrome. Journal of Endocrinological Investigation 202144 505-513. (https://doi.org/10.1007/s40618-020-01332-3)

52 Guthrie JR, Dennerstein L, Taffe JR, Lehert P \& Burger HG. The menopausal transition: a 9-year prospective population-based study. The Melbourne Women's Midlife Health Project. Climacteric 20047 375-389. (https://doi.org/10.1080/13697130400012163)

53 Davis SR, Davison SL, Donath S \& Bell RJ. Circulating androgen levels and self-reported sexual function in women. JAMA 2005294 91-96. (https://doi.org/10.1001/jama.294.1.91)

54 Santoro N, Torrens J, Crawford S, Allsworth JE, Finkelstein JS, Gold EB, Korenman S, Lasley WL, Luborsky JL, McConnell D, et al. Correlates of circulating androgens in mid-life women: the study of women's health across the nation. Journal of Clinical Endocrinology and Metabolism 2005 90 4836-4845. (https://doi.org/10.1210/jc.2004-2063)

55 Behboodi Moghadam Z, Fereidooni B, Saffari M \& Montazeri A. Measures of health-related quality of life in PCOS women: a systematic review. International Journal of Women's Health 201810 397-408. (https://doi.org/10.2147/IJWH.S165794)

56 Bazarganipour F, Ziaei S, Montazeri A, Foroozanfard F, Kazemnejad A \& Faghihzadeh S. Health-related quality of life in patients with polycystic ovary syndrome (PCOS): a model-based study of predictive factors. Journal of Sexual Medicine 201411 1023-1032. (https://doi. org/10.1111/jsm.12405)

57 Baumeister RF, Campbell JD, Krueger JI \& Vohs KD. Does high selfesteem cause better performance, interpersonal success, happiness, or healthier lifestyles? Psychological Science in the Public Interest 20034 1-44. (https://doi.org/10.1111/1529-1006.01431)

58 Rehbein-Narvaez R, Garcia-Vazquez E \& Madson L. The relation between self-esteem and sexual functioning in collegiate women. Journal of Social Psychology 2006146 250-252. (https://doi. org/10.3200/SOCP.146.2.250-252)

59 Lenhart A, Naliboff B, Shih W, Gupta A, Tillisch K, Liu C, Mayer EA \& Chang L. Postmenopausal women with irritable bowel syndrome (IBS) have more severe symptoms than premenopausal women with IBS. Neurogastroenterology and Motility 202032 e13913. (https://doi. org/10.1111/nmo.13913)

60 Bazarganipour F, Ziaei S, Montazeri A, Foroozanfard F, Kazemnejad A \& Faghihzadeh S. Body image satisfaction and self-esteem status among the patients with polycystic ovary syndrome. Iranian Journal of Reproductive Medicine 201311 829-836.

Received in final form 25 May 2021

Accepted 28 July 2021

Accepted Manuscript published online 28 July 2021 https://ec.bioscientifica.com https://doi.org/10.1530/EC-21-0257 (c) 2021 The authors Published by Bioscientifica Ltd

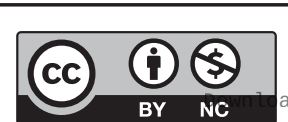

This work is licensed under a Creative Commons Attribution-NonCommercial 4.0 International License. ded from Bioscientifica.com at 04/26/2023 10:27:00AM 